\title{
BMJ Open Enhancing self-care adherence in patients with heart failure: a study protocol for developing a theory-based behaviour change intervention using the COM-B behaviour model (ACHIEVE study)
}

Oliver Rudolf Herber, ${ }^{1}$ Louise Atkins, ${ }^{2}$ Stefan Störk, ${ }^{3}$ Stefan Wilm ${ }^{1}$

To cite: Herber OR, Atkins L, Störk S, et al. Enhancing self-care adherence in patients with heart failure: a study protocol for developing a theory-based behaviour change intervention using the COM-B behaviour model (ACHIEVE study). BMJ Open 2018;8:e025907. doi:10.1136/ bmjopen-2018-025907

- Prepublication history for this paper is available online. To view these files, please visit the journal online (http://dx.doi. org/10.1136/bmjopen-2018025907).

Received 7 August 2018 Revised 13 August 2018 Accepted 14 August 2018

Check for updates

(c) Author(s) (or their employer(s)) 2018. Re-use permitted under CC BY-NC. No commercial re-use. See rights and permissions. Published by BMJ.

${ }^{1}$ Institute of General Practice (ifam), Medical Faculty of the Heinrich Heine University Düsseldorf, Düsseldorf, Germany ${ }^{2}$ UCL Centre for Behaviour Change, University College London, London, UK

${ }^{3}$ Division of Cardiology at the Outpatient Clinic of Medical Department, Comprehensive Heart Failure Center (CHFC), University Hospital Würzburg, Würzburg, Germany

Correspondence to Dr Oliver Rudolf Herber; Oliver.Herber@med.uniduesseldorf.de

\section{ABSTRACT \\ Introduction Although international guidelines} recommend self-care as an integral part of routine heart failure management, and despite evidence supporting the positive outcomes related to self-care, patients are frequently unable to adhere. Self-care can be modified through behaviour change interventions (BCls). However, previous self-care interventions have shown limited success in improving adherence to self-care, because they were neither theory-based nor well defined, which precludes the identification of underlying causal mechanisms as well as reproducibility of the intervention. Thus, our aim is to develop an intervention manual that contains theory-based BCls that are well-defined using eight descriptors proposed to describe BCls in a standardised way.

Methods and analysis BCls will be based on statements of findings derived through qualitative meta-summary techniques and a quantitative meta-analysis. These reviews will be used to extract factors (target behaviours) associated with self-care adherence/non-adherence. Extracted target behaviours will be mapped onto the 'Capability, Opportunity, Motivation and Behaviour' (COM-B) model to capture the underlying mechanisms involved. To develop approaches for change, the 'Taxonomy of Behaviour Change Techniques' will be used to allow effective mapping of the target behaviours onto established behaviour change techniques. Suggested $\mathrm{BCls}$ will then be translated into locally relevant interventions using the Normalisation Process Theory to overcome the difficulties of implementing theoretically derived interventions into practice. Finally, a consensus development method will be employed to fine-tune the content and acceptability of the intervention manual to increase the likelihood of successfully piloting and implementing future BCls into the German healthcare system.

Ethics and dissemination This study has been reviewed and approved by the Ethics Committee of the Medical Faculty of the Heinrich Heine University Düsseldorf, Germany (Ref \#: 2018-30). The results will be disseminated via peer-reviewed journal publications,
Strengths and limitations of this study

- Behaviour change intervention $(\mathrm{BCl})$ is based on sound theoretical framework.

- Extensive consultation with potential key stakeholders, that is, end users and healthcare providers, will inform intervention development.

- Final intervention manual will contain specific information regarding eight descriptors suggested for the standardised description of $\mathrm{BCls}$ to allow for replication.

- Full randomised controlled trial (RCT) design not possible within the study budget and time frame, but study will collect important data to inform a fullscale RCT in the future.

conference presentations and stakeholder engagement activities.

Trial registration number DRKS00014855; Pre-results.

\section{INTRODUCTION}

Heartfailure (HF) is a major clinical and public health problem worldwide associated with significant healthcare expenditure, morbidity and mortality. ${ }^{1}$ In Germany, the prevalence of $\mathrm{HF}$ is estimated at $3 \%-4 \%$ among adults and increases steeply with age. ${ }^{1}$ Heart failure is the most common cause of hospital admissions of patients and ranks third as cause of death. ${ }^{1}$ Treatment costs are strongly driven by expenditure for repeated hospitalisations and increase with disease progression. ${ }^{2}$ Thus, HF represents a growing public health and health economic issue as the population ages. On an individual level, patients with HF experience physical and psychological distress such as fatigue, dyspnoea, pain, depression or problems regarding concentration, all of 
which are having a major negative impact on the person's quality of life. $^{3}$

After being diagnosed with HF, the prognosis depends on two major components: one is physician-based and encompasses all aspects of appropriate treatment, monitoring of effects and communicating relevant information; the other is patient-based and refers to the concept of self-care including the motivation to adhere to diagnostic and therapeutic recommendations. HF care should be exerted in a multidisciplinary team and involves a patient-centred approach seeking to systematically develop emotional, physical, intellectual and social resources of the patient. ${ }^{4}$ Ultimately, such strategy is thought to empower patients in order to improve and sustain efficacious self-care behaviour. The term self-care refers to

the individual's ability to manage the symptoms, treatment, physical and psychosocial consequences and life style changes inherent in living with a chronic condition. Efficacious self-care encompasses the ability to monitor one's condition and to effect the cognitive, behavioural and emotional responses necessary to maintain a satisfactory quality of life. ${ }^{5}$ (p. 178)

Since 2008, the European Guidelines for Diagnosis and Treatment of HF strongly emphasise self-care (Grade IA recommendation) as part of routine HF management and patient empowerment. ${ }^{46}$

Generally, self-care mandates a sustained effort in order to impact on any chronic disease. In particular, successful HF therapy requires a substantial amount of self-care and adherence to multiple aspects of the treatment regimen. ${ }^{7}$ For example, HF self-care encompasses a complex set of behaviours including daily weighing, low-sodium diet, fluid restriction, regular physical exercise, medication taking, symptom monitoring involving exercising and developing an awareness of exacerbating symptoms such as shortness of breath, lower extremity oedema, fatigue or activity intolerance. ${ }^{8}$ Hence, such adherence to HF selfcare is considered key in order to achieve a positive effect on disease progression. Adherence is defined as the extent to which a patient's behaviour coincides with the recommendations made by healthcare providers. ${ }^{9}$ The concept of adherence focuses on specific patient behaviours emphasising the need for agreement between patient and healthcare provider. Sound evidence from systematic reviews has shown that adherence to self-care significantly reduces HF-related hospitalisations, ${ }^{10}$ morbidity and mortality, ${ }^{11}$ decreases hospital readmissions ${ }^{12}$ and improves HF-related quality of life. ${ }^{13}$ Conversely, failure to adhere to recommended self-care is associated with $\mathrm{HF}$ exacerbation, ${ }^{14}$ frequent hospital readmissions and low survival. ${ }^{15}$

Patients with HF are faced with the difficulty of adopting complex recommendations including adherence to multiple aspects of the treatment regimen and lifestyle adjustments in order to prevent disease progression. Self-management interventions aiming at improving self-care generally seek to empower individuals by endowing them with the skills necessary to actively participate in the management of their chronic condition. ${ }^{5}$ Several systematic reviews investigating the efficacy and effectiveness of self-management interventions involving home and/or clinic visits, education sessions (eg, 'giving information'), telemonitoring approaches and follow-up telephone calls for patients with HF have been published to date showing inconsistent results. Earlier conducted systematic reviews had suggested a relevant benefit in relation to hard clinical endpoints including all-cause hospitalisation and HF-related hospitalisations. ${ }^{12}{ }^{16}$ Despite favourable pooled effects, some more recently conducted large randomised controlled trials (RCTs) including multisite studies showed inconsistent results. ${ }^{17-20}$ Thus, the effectiveness of self-management interventions in terms of all-cause hospitalisation, HF-related hospitalisations and death remains inconclusive until today. Part of this heterogeneity may be attributable to the absence of self-management interventions applying behaviour strategies and/or cognitive behavioural strategies (rather than simply educational interventions), insufficient use of theoretical models underpinning the intervention or lack of detailed description of the active ingredient(s) driving the intervention.

In more detail, evidence of grade A (high quality) exists that interventions using established, well-defined behaviour change techniques (ie, the 'active ingredient' of an intervention) are most effective. ${ }^{21}$ Yet, a review of self-management interventions promoting self-care of patients with HF came to the conclusion that only very few studies used explicit theory-based interventions, while the majority still lacked specificity of the mechanisms employed to improve self-care. ${ }^{22}$ Such critique was also articulated by Michie and colleagues ${ }^{23}$ who stated that interventions are commonly designed without applying an evidence-based underlying model. Instead, interventions are frequently based on implicit common sense models. ${ }^{24}$ Similarly, a most recent critical reflection on methodological challenges encountered in meta-analyses on self-care interventions advised intervention designers to pay particular attention to the causal mechanisms underlying the intervention when designing studies. ${ }^{25}$ Hence, absent effects of previous self-management interventions seeking to improve self-care may very well be attributable to the lack of theoretical underpinning or the use of ill-chosen strategies. ${ }^{26} 27$

Another criticism relates to the lack of detailed description of the active ingredient(s) driving a successful intervention. Detailed descriptions are of paramount importance for replication, allowing scientists to accumulate evidence about intervention effects and causal mechanisms. ${ }^{28}$ For the results to be judged, reproducible, sufficiently detailed information must be provided to allow the potential for reproducing the same or similar results. ${ }^{29}$ Yet, specific features rendering interventions successful are often ill-defined in research publications, which limit reproducibility. ${ }^{18}$ Hence, a systematic method 
that incorporates a thorough understanding of the nature of the behaviour to be changed (ie, the target behaviour), an appropriate system for characterising interventions and their components as well as descriptors suggested for the standardisation of reporting behaviour change interventions (BCIs) are mandatory. In this way, a 'science of change' can be established in which effective interventions can be understood and replicated. ${ }^{30}$

In summary, it can be stated that inconclusive results of existing self-management interventions for patients with HF may very well be attributable to the absence of interventions applying behaviour strategies and/or cognitive behavioural strategies, insufficient use of theoretical models underpinning the intervention or lack of detailed description of the active ingredient(s) driving the intervention, thus emphasising the need to design an intervention that allows for these likely sources of heterogeneity. Therefore, the aim of this study is to develop well-defined theory-based BCIs based on an underlying behaviour model that allows for identifying effective behaviour change techniques.

\section{METHODS AND ANALYSIS}

\section{Patient and public involvement}

Our research question emerged from the findings of published literature on self-management interventions as described above. Patients and the public are involved in this early stage of designing BCIs through their involvement as members of the participatory planning group. To facilitate successful completion of each stage of the proposed work programme as described below, a participatory planning group will be established right at the beginning of the study. Intervention development and implementation should be based on meaningful participation of key stakeholders. ${ }^{31}$ Thus, for the life of this project, a participatory planning group will be deployed to assist in the development of an intervention manual containing well-defined theory-based BCIs. The planning group will be composed of key stakeholders who have a particular interest in designing BCIs including intervention developers, intervention implementers and intervention end users (ie, patients with HF). For identifying key stakeholders, we will be using the guiding questions proposed by Preskill and Jones. ${ }^{32}$ They consider the following five domains to be relevant for the selection process: (1) expertise, (2) diverse perspectives, (3) responsibility and authority, (4) influence and (5) commitment. The participatory planning group will meet at least every 6 months or at shorter intervals, if required.

\section{Using the MRC framework for designing complex interventions}

The Medical Research Council (MRC) four-stage framework consisting of 'develop-test-evaluate-implement' will be used as an underlying framework for researching complex interventions. Before embarking on a full RCT, preparatory and exploratory studies may be required to gradually refine the study design. ${ }^{33}$ To develop an acceptable, effective and sustainable complex intervention, key elements are proposed. ${ }^{34}$ The present project proposal addresses the 'development' stage of the MRC framework to design an intervention on a theoretical base prior to its preliminary testing in an exploratory trial (figure 1).

Essentially, in the development stage, a paper-based exercise will be undertaken dealing with issues such as: the specific content of the intervention, who will deliver it, how long it will take to deliver, what each stakeholder will actually do as part of the intervention, and should the intervention prove effective, how it may be implemented into routine practice to increase the likelihood of its adoption by patients and health services. Providing robust answers to the above questions is of paramount importance to avoid what Chalmers and Glasziou ${ }^{35}$ referred to as 'research waste', that is, research where insufficient effort has been made to develop and pilot test the intervention before proceeding to a full RCT. However, since the MRC framework provides no specific guidance on how to link theory and intervention techniques, the Capability, Opportunity, Motivation (COM-B) model of behaviour ${ }^{23}$ in combination with the Taxonomy of Behaviour Change Techniques $^{36}$ will be employed. To our knowledge, COM-B is the only model that provides a systematic and transparent way of identifying target behaviours and techniques judged to be most effective in changing behaviour. The COM-B model has been applied successfully in intervention development in various health-related contexts including changing eating behaviours, ${ }^{37}$ enhancing medication adherence, ${ }^{38}$ reducing blood pressure-related disease burden ${ }^{39}$ or improving hearing aid use in adults. ${ }^{40}$ To manage the process of developing BCIs in a systematic fashion, our work programme is guided by four stages following intervention mapping. ${ }^{41}$

\section{Stage 1: systematic reviews for extracting desirable/undesirable} behaviours

Based on the findings from our recently completed systematic review and qualitative meta-summary, ${ }^{42} 37$ statements of findings pertaining to self-care were identified representing a comprehensive inventory of findings across 31 qualitative (QUAL) reports. However, the evidence from qualitative enquiries alone might not be satisfactory for designing sound evidence-based interventions. Thus, in addition to drawing on our qualitative synthesis we have identified a comprehensive systematic review and meta-analysis (QUAN) based on 65 reports describing the current evidence concerning determinants of self-care in patients with $\mathrm{HF}^{43}$ Hence, two up-to-date comprehensive reviews synthesising qualitative and quantitative studies will be used to first identify and then extract all factors (target behaviours) associated with self-care adherence/ non-adherence. Two members of staff will identify and extract all behaviours associated with adherence/non-adherence to HF self-care independently from each other and then compare notes to create a final list of common behaviours from both reviews (QUAN+QUAL). At the 


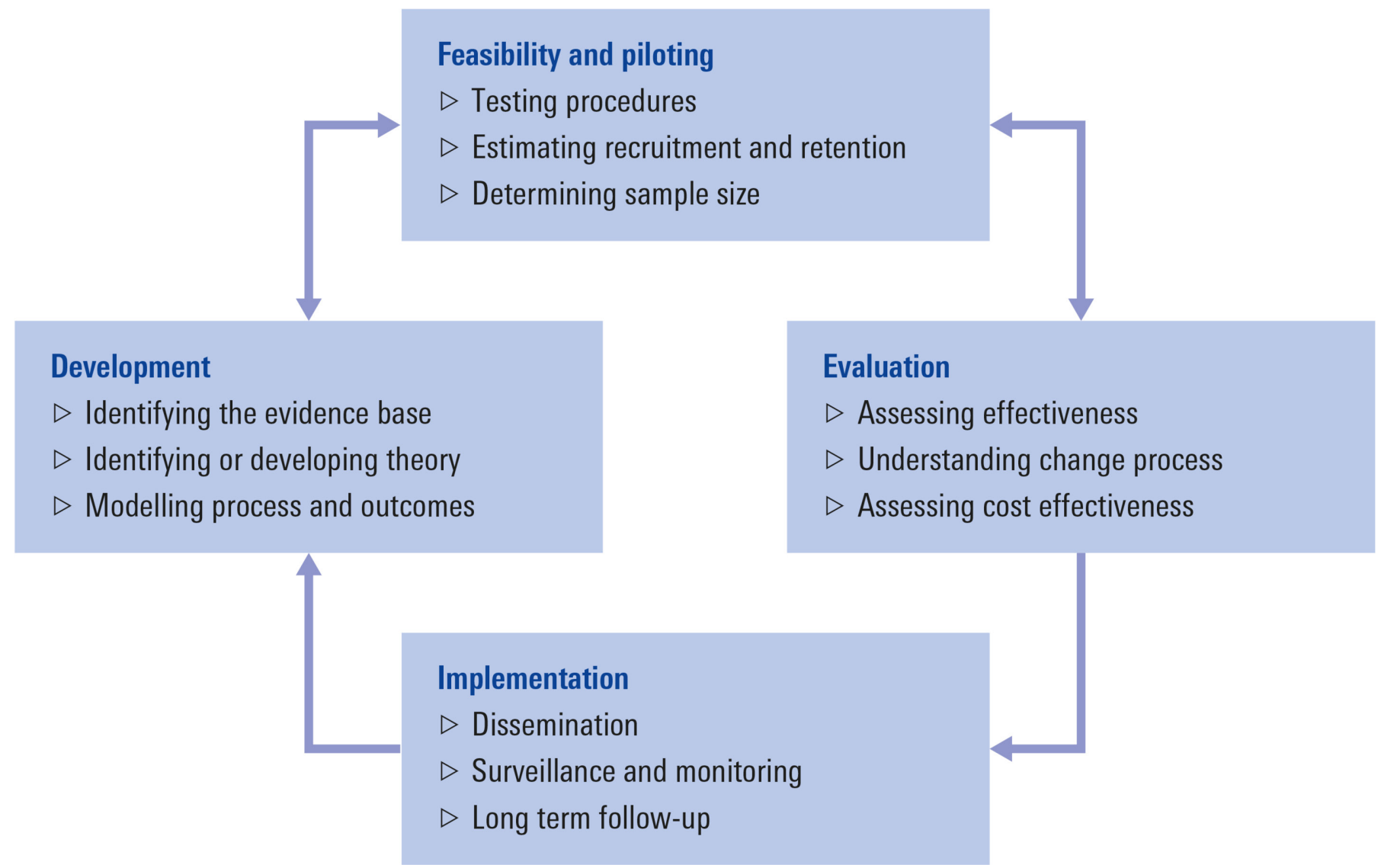

Figure 1 Key elements of the MRC framework for developing complex interventions. ${ }^{38}$ MRC, Medical Research Council.

end of stage 1, a final list of common factors extracted is produced in order to map them onto the COM-B model of behaviour (see stage 2 below).

\section{Stage 2: mapping of extracted behaviours onto the COM-B} behaviour model

The application of underlying behavioural theories is vital for designing BCIs, ${ }^{44}$ because theory-driven interventions are considered superior. ${ }^{45}$ Because of its successful utilisation in intervention development, the COM-B model will be used as a universal behavioural theory to enhance the understanding of causal mechanisms underlying behaviour change (figure 2). The COM-B model will allow a more fine-grained analysis of the causes of adherence/non-adherence, so that an intervention can be selected more precisely to target a particular behaviour. The COM-B model in combination with the 'Behaviour Change Wheel' ${ }^{23}$ is the centrepiece of designing BCIs. The behaviour change wheel-consisting of three concentric circles-will aid in visualising how intrapersonal and/or interpersonal behaviour can be changed by targeting specific underlying modifiable mechanisms (figure 3). Thus, each of the factors identified in stage 1 will be mapped onto the COM-B model (equals inner circle of the wheel) using the definitions regarding components and subcomponents as described below. If there are difficulties in classifying the factors onto the COM-B model, a second opinion will be obtained until a clear assignment can be made. We are aware that not all factors identified from the literature review will fit into exactly one subcomponent since the effects of the factors might work via a number of components. ${ }^{38}$ This, however, should not be considered as a limitation of the model because the effects of the factors are explained by components within the model. For quality assurance, all factors mapped onto the COM-B model will be double-checked for accuracy by a research team member not involved in the mapping exercise.

The COM-B model assists in understanding behaviour and as such will provide an explanation for why patients with $\mathrm{HF}$ adhere and/or non-adhere to self-care. The COM-B model consists of three components: (1) capability, (2) opportunity and (3) motivation (COM) that causes the performance of behaviour (B) and in turn influences these three components.

To capture important distinctions, the three components are further divided into two subcomponents each. 'Capability' is subdivided into psychological capability (the capacity to engage in the necessary thought processes) and physical capability (the capacity to engage in necessary physical processes). ${ }^{23}$ Psychological capability includes the comprehension of the disease and its treatment, cognitive functioning such as the capacity for judgement, thinking or memory as well as executive function like the capacity to plan. ${ }^{38}$ Physical capability involves the capacity to adapt to lifestyle changes such as 


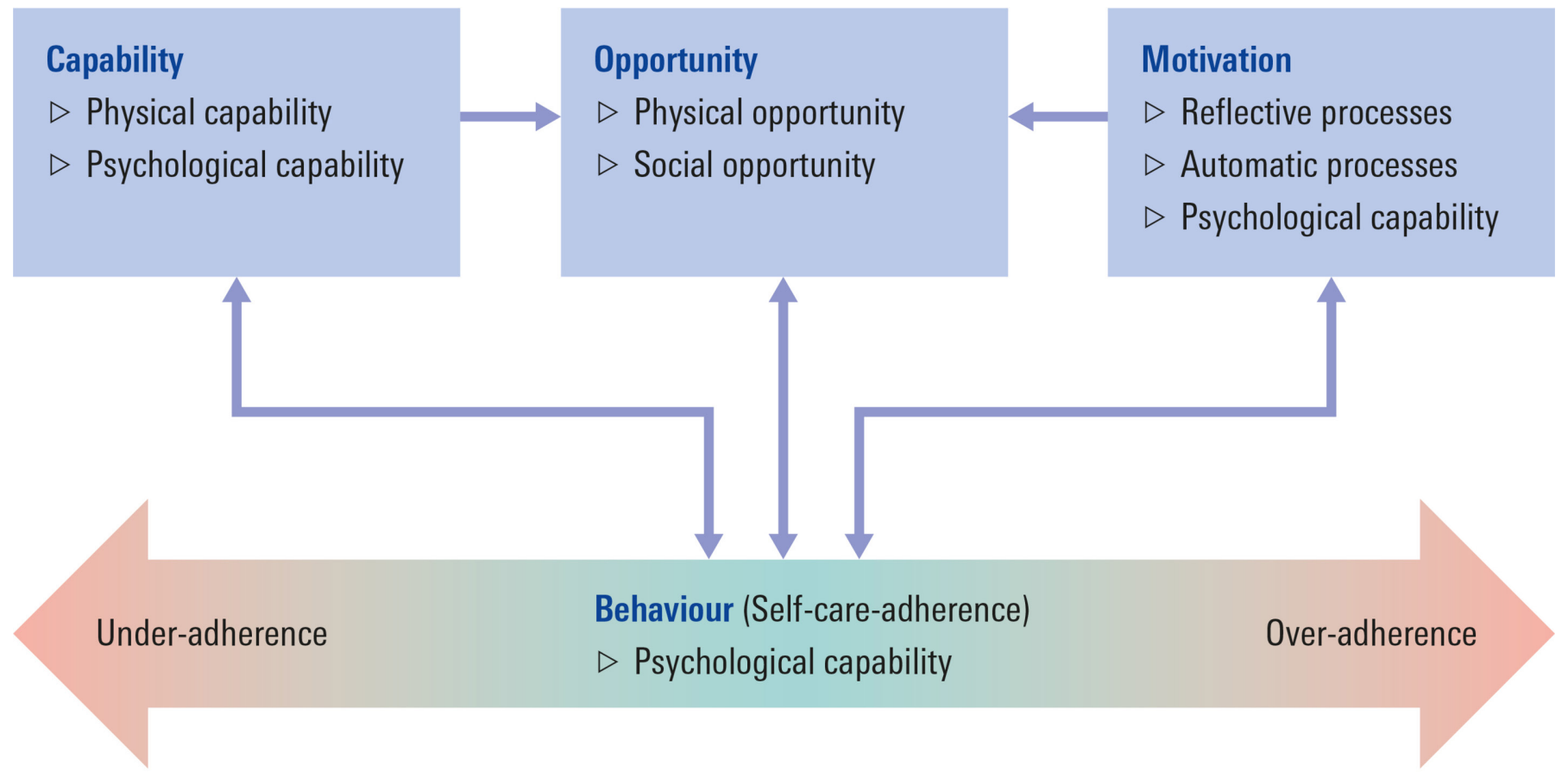

Figure 2 Application of the COM-B model to self-care adherence. ${ }^{42} \mathrm{COM}-\mathrm{B}$, Capability, Opportunity, Motivation and Behaviour.

a diet or social behaviours as well as dexterity. ${ }^{38}$ 'Opportunity' is subdivided into physical opportunity (provided by the environment) and social opportunity (provided by the cultural milieu that dictates the way we think about things) ${ }^{23}$ Physical opportunity includes cost, access (eg, availability of a weighing scale), regimen complexity, social support as well as healthcare professional-patient relationship and communication. ${ }^{38}$ Social opportunity comprises the stigma of the disease, the fear of disclosure as well as religious and/or cultural beliefs. ${ }^{38}$ 'Motivation' is subdivided into reflective processes (involving plans and evaluations) and automatic processes (involving emotions and impulses that arise from associative learning and/or innate dispositions). ${ }^{23}$ Reflective motivation entails the perception of the illness (eg, cause, chronic vs acute), beliefs about treatment (eg, necessity, efficacy, concerns

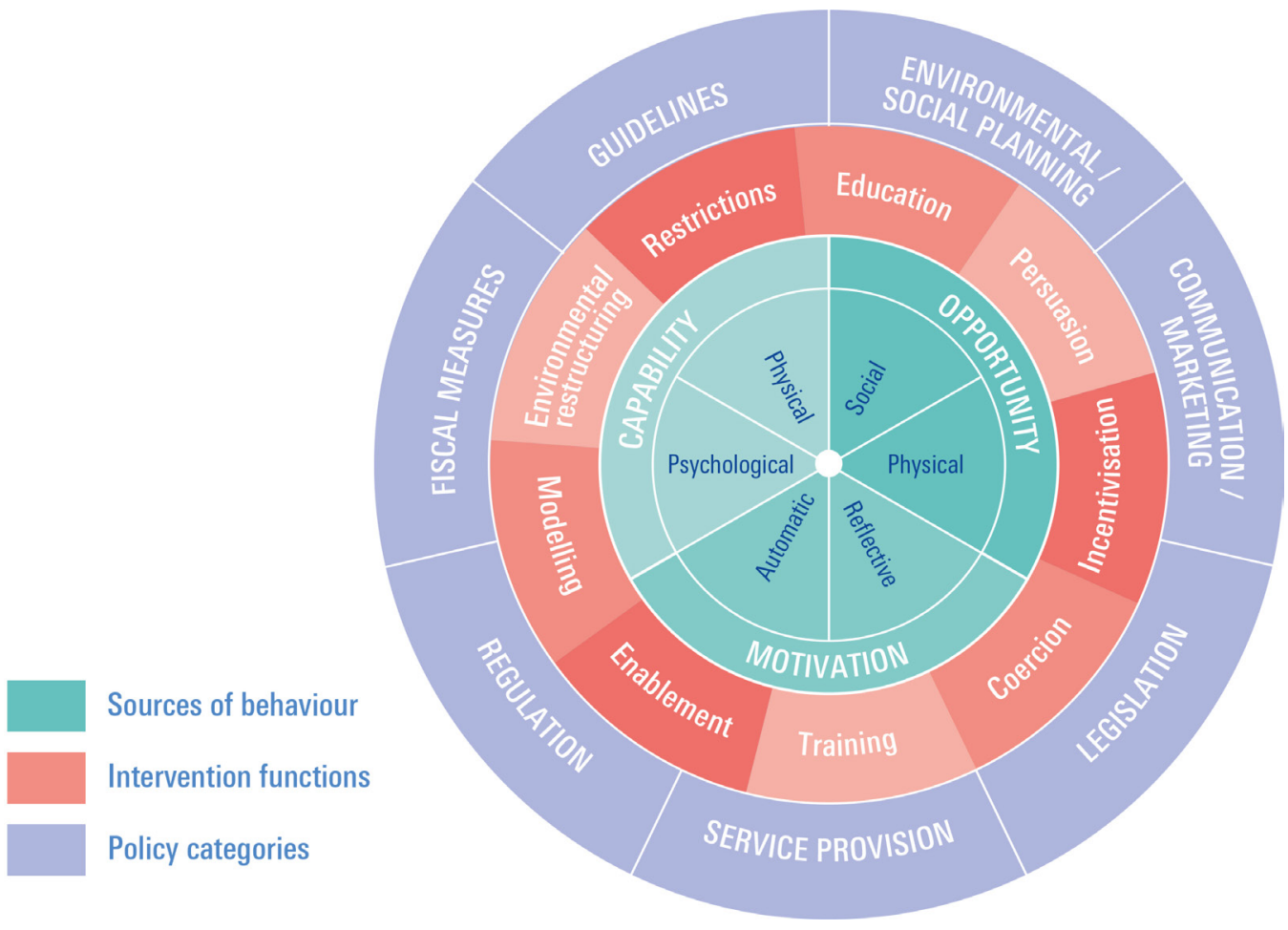

Figure 3 The behaviour change wheel. ${ }^{23} 30$ 
about current or future adverse events), outcome expectancies as well as self-efficacy. ${ }^{38}$ Automatic motivation contains stimuli or cues for action as well as mood state or mood disorder such as depression and anxiety. ${ }^{38}$ At the end of stage 2, all factors (target behaviours) are mapped onto the COM-B model in order to identify behaviour change techniques appropriate for modifying behaviours (see stage 3 below).

\section{Stage 3: mapping of target behaviours onto behaviour change techniques}

In the next step, we will identify behaviour change techniques that are appropriate for changing undesirable behaviours associated with self-care non-adherence or to reinforce desirable behaviours associated with selfcare adherence using the behaviour change wheel (figure 3). The middle circle of the 'behaviour change wheel' contains a choice of nine evidence-based intervention functions that are aimed at addressing the target behaviours identified with the help of the COM-B model in stage 2. Please note that the outer circle of the wheel represents policy categories that will apply less to behavioural change in the individual.

In order to develop approaches for change, the Taxonomy of Behaviour Change Techniques (BCTs) developed by Michie et $a l^{3644}$ or the concepts described by Bartholomew et $a t^{41}$ will be used to allow effective mapping of factors onto behaviour change techniques to address specific behavioural determinants. According to Michie, ${ }^{46}$ a BCT is defined as

an active component of an intervention designed to change behaviour. The defining characteristics of a BCT are that it is 'observable, replicable, irreducible', a component of an intervention designed to change behaviour and a postulated active ingredient within the intervention. It is thus the smallest component compatible with retaining the postulated active ingredients, that is, the proposed mechanisms of change, and can be used alone or in combination with other BCTs. (p. 182)

The Taxonomy of BCTs was developed in a series of consensus exercises involving over 50 behaviour change experts from various disciplines and countries. It contains 93 itemised health BCTs that are clustered into 16 groupings; each group containing between 3 and 11 reliable, distinct BCTs. This cross-domain taxonomy applies to a wide range of behaviours and various types of interventions and enjoys international acceptance and use. We will use this taxonomy for the systematic specification of BCIs. For example, if a patient has difficulties interpreting his or her symptoms or attributes them to existing comorbidities, medication side effects or emotional responses, then a technique that targets to change psychological capability should be selected. A respective intervention might involve techniques such as shaping knowledge or feedback and monitoring. ${ }^{36}$ The full range of all BCTs available is depicted by Michie $e t a \hat{l}^{6}$ including examples of how BCTs can be implemented and information on how to operationalise BCTs. At the end of stage 3, BCTs are formulated in order to identify relevant factors that allow its successful implementation into routine clinical work (see stage 4 below).

Stage 4: identifying wider factors that influence successful implementation of $\mathrm{BCls}$ into practice

This stage involves the consultation of intended participants and implementers to identify wider relevant factors needed for successful implementation of BCIs into routine work. Suggested BCIs will be translated into locally relevant interventions using the Normalisation Process Theory (NPT) to overcome the difficulties of implementing theoretically derived interventions into everyday practice. ${ }^{478}$ NPT will be used to identify factors that individuals and groups need to do in order to enable true integration of BCIs designed in stage 3 into routine work. ${ }^{49}$ To promote adoption and faithful delivery of our interventions in the future, it is crucial to involve allstakeholders in determining the intervention content and the best way to deliver it. ${ }^{50}$ NPT will provide guiding questions for qualitative interviews with key stakeholders that will shed light on a range of relevant contextual issues ${ }^{48}$ necessary to increase practical effectiveness, that is, whether the intervention will work in clinical practice. ${ }^{51}$ Consequently, NPT assists in better defining the core components of the intervention and to examine acceptability to patients, their families and health professionals.

Qualitative semistructured interviews with approximately 15-17 key stakeholders, that is, those targeted by the intervention or involved in its development or delivery, will be conducted using NPT to guide the interview questions. Potential participants (eg, patients with HF, health professionals, academics and so on) will be identified by the participatory planning group (see above) to ensure that all relevant stakeholders are represented and their views and preferences are sought. Participants will be contacted by the researcher to arrange for the interview. Interviews will be conducted in places that are convenient for the stakeholders according to their preferences. As described by Lovell $e t a l,{ }^{52}$ guiding interview questions will cover the four components of NPT, that is, (1) questions considering meaning and sense making by stakeholders (coherence); (2) commitment and engagement by stakeholders (cognitive participation); (3) the work stakeholders do to make the intervention function (collective action) and (4) stakeholders appraising the intervention (reflexive monitoring). In addition, interviews will allow reflection on, and refinement of, content and acceptability of BCTs as well as specific information regarding the following eight descriptors suggested for the standardised description of BCIs: (1) content or elements of the intervention; (2) characteristics of self-care tutors (eg, health professionals, lay tutors); (3) characteristics of the target population (eg, adults, children); (4) delivery location (eg, hospital, GP practice, home environment); (5) mode 
of delivery (eg, group-based, individual approach); (6) format (eg, lectures, manual); (7) intensity (eg, contact time) and (8) duration (eg, number of sessions over a given period) ${ }^{53}$

Participating key stakeholders will be asked for consent to the interview. All interviews will be audio recorded and transcribed verbatim; specific details will be anonymised. Qualitative data will be analysed using thematic analysis. ${ }^{54}$ To assure validity, interview texts will be analysed in a multidisciplinary team by reading the transcripts and identifying emerging themes and categories. Analysis of the data will be informed by the aforementioned four components of the NPT framework. Regarding the eight descriptors, in addition to obtaining information from our stakeholders through qualitative enquiry, we will also retrieve and summarise, if available, specific HF self-care-related evidence from previously published quantitative reviews. For example, is there any evidence that supports the effectiveness of either group-based or individual approaches (mode of delivery) in enhancing self-care in patients with HF? The results of the reviews, if any, will be combined with the individual responses from the key stakeholders on a matrix; any ambiguities will be addressed with the help of a consensus development method (Delphi technique) in order to achieve clarity concerning the descriptors.

Finally, the Delphi technique-a formal consensus development research method-involving all key stakeholders will be used to elicit consensus. All stakeholders will receive an invitation to participate, detailing the rationale for the consensus exercise. A matrix of the combined results and a copy of the Delphi questionnaire will be circulated. Stakeholders who will not respond within 4 weeks will receive a follow-up invitation. The matrix will serve as a platform for discussion to derive the final intervention manual. The Delphi questionnaire will deal specifically with the mixed responses (ambiguities) that remain regarding the descriptors. The process of collating and distributing an anonymised summary of the stakeholders' responses and adjusting earlier answers based on feedback from the group will be repeated for a maximum of three rounds. In line with the recommendations by Diamond et al, ${ }^{55}$ consensus will be a priori operationalised as a proportion of participants agreeing to a particular descriptor. For example, the threshold for determination of consensus might be set at $75 \%$ of participating stakeholders. ${ }^{56}$ If, however, consensus values for certain descriptors will remain below the prespecified threshold after the third iteration, a rank order will be used instead giving preference to the descriptor with the highest degree of agreement. In summary, the Delphi technique will aid in fine-tuning our BCIs to increase the likelihood of its acceptance. At the end of stage 4, we will have produced a final version of the intervention manual containing well-defined theory-based BCIs including the concept and outline of an exploratory trial ready for pilot testing in a subsequent full randomised controlled intervention trial.

\section{DISCUSSION}

The main outcome of this study is to produce a detailed intervention manual that contains well-defined (ie, using the eight descriptors suggested for the standardised description of interventions) theory-based (ie, based on the COM-B behaviour model) BCIs. Following the MRC framework for designing complex interventions, the envisaged subsequent studies encompass: (1) the execution of an exploratory trial, and if feasible, (2) the completion of a full RCT. Within the scope of an exploratory trial, the feasibility and acceptance of the actual intervention described in the manual will be pilot tested. Particular emphasis will be placed on recruitment strategies, estimates of recruitment numbers, collaborating institutions or issues concerning the identification of specific outcome measures for a full RCT. Finally, an economic evaluation in the form of a cost-effectiveness analysis will be conducted as part of evaluating the BCIs compared with an appropriate alternative. Therefore, the design of a health economic evaluation (eg, cost-effectiveness analysis, cost-utility analysis) from both a societal and a statutory health insurance perspective will be developed and appropriate measures for effects and costs defined as well as instruments for data collection.

\section{Strengths and limitations of this study}

All BCIs are based on a sound theoretical framework. Besides that, continuous consultation with potential key stakeholders, that is, end users and healthcare providers, will inform intervention development to ensure successful implementation of BCIs into practice. Furthermore, the final intervention manual will contain specific information regarding the eight descriptors suggested for the standardised description of BCIs to allow for replication. However, the execution of a full RCT design is not possible within the study budget and time frame, but this current study will collect important data to inform a fullscale RCT in the future.

\section{Ethics and dissemination}

The findings of the study will be disseminated through peer-reviewed journal articles, national and international conference presentations and stakeholder engagement activities (ie, via our participatory planning group members).

Contributors ORH: designed the study and drafted the manuscript. SS, LA and SW: provided valuable input in designing the study and critically reviewed and edited the manuscript. All authors read and approved the final manuscript.

Funding This work was supported by the German Research Foundation (Deutsche Forschungsgemeinschaft, DFG); grant number HE 7352/1-2.

Disclaimer This study's contents are solely the responsibility of the authors and do not necessarily represent the official view of DFG. The funding body had no involvement in the design of the study and will not be involved in the conduct of the study including data collection, analysis and interpretation of data and in writing manuscripts for publication.

Competing interests None declared.

Ethics approval Ethics Committee of the Medical Faculty of Heinrich Heine University, Düsseldorf, Germany (Ref \#: 2018-30). 
Provenance and peer review Not commissioned; peer reviewed for ethical and funding approval prior to submission.

Open access This is an open access article distributed in accordance with the Creative Commons Attribution Non Commercial (CC BY-NC 4.0) license, which permits others to distribute, remix, adapt, build upon this work non-commercially, and license their derivative works on different terms, provided the original work is properly cited, appropriate credit is given, any changes made indicated, and the use is non-commercial. See: http://creativecommons.org/licenses/by-nc/4.0/.

\section{REFERENCES}

1. Störk S, Handrock R, Jacob J, et al. Epidemiology of heart failure in Germany: a retrospective database study. Clin Res Cardiol 2017;106:913-22.

2. Schmid T, Xu W, Gandra SR, et al. Erratum to: 'Costs of treating cardiovascular events in Germany: a systematic literature review'. Health Econ Rev 2016;6:1.

3. Li CC, Shun SC. Understanding self care coping styles in patients with chronic heart failure: a systematic review. Eur J Cardiovasc Nurs 2016;15:1.

4. Ponikowski P, Voors AA, Anker SD, et al. ESC Guidelines for the diagnosis and treatment of acute and chronic heart failure: the task force for the diagnosis and treatment of acute and chronic heart failure of the european society of cardiology (esc) developed with the special contribution of the heart failure association (HFA) of the ESC. Eur Heart J 2016;2016:27.

5. Barlow J, Wright C, Sheasby J, et al. Self-management approaches for people with chronic conditions: a review. Patient Educ Couns 2002;48:177-87.

6. Lainscak M, Blue L, Clark AL, et al. Self-care management of heart failure: practical recommendations from the patient care committee of the heart failure association of the european society of Cardiology. Eur J Heart Fail 2011;13:115-26.

7. Hammash MH, Crawford T, Shawler C, et al. Beyond social support: Self-care confidence is key for adherence in patients with heart failure. Eur J Cardiovasc Nurs 2017;16:632-7.

8. Yancy CW, Jessup M, Bozkurt B, et al. 2013 ACCF/AHA guideline for the management of heart failure: executive summary: a report of the American College of Cardiology Foundation/American Heart Association Task Force on practice guidelines. Circulation 2013;128:1810-52.

9. Jaarsma T, Nikolova-Simons M, van der Wal MH. Nurses' strategies to address self-care aspects related to medication adherence and symptom recognition in heart failure patients: an in-depth look. Heart Lung 2012;41:583-93.

10. McAlister FA, Stewart S, Ferrua S, et al. Multidisciplinary strategies for the management of heart failure patients at high risk for admission: a systematic review of randomized trials. J Am Coll Cardiol 2004;44:44:4.

11. Albert NM. Promoting self-care in heart failure: state of clinical practice based on the perspectives of healthcare systems and providers. J Cardiovasc Nurs 2008;23:3.

12. Jovicic A, Holroyd-Leduc JM, Straus SE. Effects of self-management intervention on health outcomes of patients with heart failure: a systematic review of randomized controlled trials. BMC Cardiovasc Disord 2006;6:43.

13. Jonkman $\mathrm{NH}$, Westland $\mathrm{H}$, Groenwold $\mathrm{RH}$, et al. Do self-management interventions work in patients with heart failure? An individual patient data meta-analysis. Circulation 2016;133:12.

14. Hunt SA, Abraham WT, Chin MH, et al. Focused update incorporated into the ACC/AHA 2005 guidelines for the diagnosis and management of heart failure in adults. A report of the American College of Cardiology Foundation/American heart association task force on practice guidelines developed in collaboration with the international society for heart and lung transplantation. J Am Coll Cardiol 2009;2009:e1-e90.

15. JR W, Moser DK, Chung ML, et al. Objectively measured, but not self-reported, medication adherence independently predicts eventfree survival in patients with heart failure. J Card Fail 2008;14:3.

16. Ditewig JB, Blok H, Havers J, et al. Effectiveness of self-management interventions on mortality, hospital readmissions, chronic heart failure hospitalization rate and quality of life in patients with chronic heart failure: a systematic review. Patient Educ Couns 2010;78:297-315.

17. Dracup K, Moser DK, Pelter MM, et al. Randomized, controlled trial to improve self-care in patients with heart failure living in rural areas. Circulation 2014;130:256-64.

18. Angermann CE, Störk S, Gelbrich G, et al. Mode of action and effects of standardized collaborative disease management on mortality and morbidity in patient with systolic heart failure. Circ Heart Fail 2012;5:1.

19. DeWalt DA, Schillinger D, Ruo B, et al. Multisite randomized trial of a single-session versus multisession literacy-sensitive self-care intervention for patients with heart failure. Circulation 2012;125:2854-62.

20. Powell LH, Calvin JE, Richardson D, et al. Self-management counseling in patients with heart failure: the heart failure adherence and retention randomized behavioral trial. JAMA 2010;304:12.

21. Greaves CJ, Sheppard KE, Abraham C, et al. Systematic review of reviews of intervention components associated with increased effectiveness in dietary and physical activity interventions. BMC Public Health 2011;11:119.

22. Barnason S, Zimmerman L, Young L. An integrative review of interventions promoting self-care of patients with heart failure. J Clin Nurs 2012;21:448-75.

23. Michie S, van Stralen MM, West R. The behaviour change wheel: a new method for characterising and designing behaviour change interventions. Implement Sci 2011;6:42.

24. Michie S, Fixsen D, Grimshaw JM, et al. Specifying and reporting complex behaviour change interventions: the need for a scientific method. Implement Sci 2009;4:40.

25. Jonkman NH, Groenwold RHH, Trappenburg JCA, et al. Complex self-management interventions in chronic disease unravelled: a review of lessons learned from an individual patient data metaanalysis. J Clin Epidemiol 2017;83:48-56.

26. Patton DE, Hughes CM, Cadogan CA, et al. Theory-based interventions to improve medication adherence in older adults prescribed polypharmacy: a systematic review. Drugs Aging 2017;34:2:97-113.

27. van Achterberg T. How to arrive at an implementation plan. In: Richards DA, Hallberg IR, eds. Complex interventions in health: An overview of research methods. Oxon: Routledge, 2015:282-92.

28. Michie S, Abraham C. Advancing the science of behaviour change: a plea for scientific reporting. Addiction 2008;103:9.

29. Halsey LG, Curran-Everett D, Vowler SL, et al. The fickle $P$ value generates irreproducible results. Nat Methods 2015;12:179-85.

30. Michie S, Atkins L, West R. The behaviour change wheel - a guide to designing interventions. Silverback Publishing, 2014.

31. Centre for Disease Control. Program performance and evaluation office (PPEO) - Program evaluation. 2012 https://www.cdc.gov/ EVAL/framework/ (accessed 16 March 2017).

32. Preskill H, Jones N. A practical guide for engaging stakeholders in developing evaluation questions. Princeton: NJ: Robert Wood Johnson Foundation, 2009.

33. Craig P, Dieppe P, Macintyre S, et al. Medical Research Council Guidance. Developing and evaluating complex interventions: the new Medical Research Council guidance. BMJ 2008;337:a1655.

34. Medical Research Council. Developing and evaluating complex interventions: new guidance. London: Medical Research Council, 2006.

35. Chalmers I, Glasziou P. Avoidable waste in the production and reporting of research evidence. Lancet 2009;374:86-9.

36. Michie S, Richardson M, Johnston M, et al. The behavior change technique taxonomy (v1) of 93 hierarchically clustered techniques: building an international consensus for the reporting of behavior change interventions. Ann Behav Med 2013;46:81-95.

37. Atkins L, Michie S. Designing interventions to change eating behaviours. Proc Nutr Soc 2015;74:164-70.

38. Jackson C, Eliasson L, Barber N, et al. Applying COM-B to medication adherence: A suggested framework for research and interventions. Eur Health Psychol 2014;16:1.

39. Peiris D, Thompson SR, Beratarrechea A, et al. Behaviour change strategies for reducing blood pressure-related disease burden: findings from a global implementation research programme. Implement Sci 2015;10:158.

40. Barker F, Atkins L, de Lusignan S. Applying the com-b behaviour model and behaviour change wheel to develop an intervention to improve hearing-aid use in adult auditory rehabilitation. Int J Audiol 2016;55(Suppl 3):S90-S98.

41. Bartholomew Eldredge LK, Markham CM, Ruiter RAC, et al. Planning health promotion programs: An intervention mapping approach. $4^{\text {th }}$ edn. San Francisco, CA: Jossey-Bass, 2016.

42. Herber OR, Bücker B, Metzendorf MI, et al. A qualitative metasummary using sandelowski and barroso's method for integrating qualitative research to explore barriers and facilitators to self-care in heart failure patients. Eur J Cardiovasc Nurs 2017;16:662-77.

43. Kessing D, Denollet J, Widdershoven J, et al. Psychological determinants of heart failure self-care: Systematic review and metaanalysis. Psychosom Med 2016;78:4. 
44. Michie S, Johnston M, Francis J, et al. From theory to intervention: Mapping theoretically derived behavioural determinants to behaviour change techniques. Appl Psychol 2008;57:660-80.

45. Jenkins A, Christensen H, Walker JG, et al. The effectiveness of distance interventions for increasing physical activity: a review. $A m \mathrm{~J}$ Health Promot 2009;24:102-17.

46. Michie S. In: Gellman MT, Rick J, editors. Encyclopedia of Behavioural Medicine: London: Springer Behaviour change techniques, 2013:182-7.

47. Murray E, Treweek S, Pope C, et al. Normalisation process theory: a framework for developing, evaluating and implementing complex interventions. BMC Med 2010;8:63.

48. May C, Finch T. Implementing, embedding, and integrating practices: an outline of normalization process theory. Sociology 2009;43:3:535-54.

49. May CR, Finch T, Ballini L, et al. Evaluating complex interventions and health technologies using normalization process theory: development of a simplified approach and web-enabled toolkit. BMC Health Serv Res 2011;11:245.

50. Abraham C, Denford S, Smith JS, et al. Designing interventions to change health-related behaviour. In: Richards DA, Hallberg IR, eds.
Complex interventions in health: An overview of research methods. Oxon: Routledge, 2015:103-10.

51. Haynes B. Can it work? Does it work? Is it worth it? The testing of healthcare interventions is evolving. BMJ 1999;319:7211.

52. Lovell K, Bower P, Richards D, et al. Developing guided selfhelp for depression using the medical research council complex interventions framework: a description of the modelling phase and results of an exploratory randomised controlled trial. BMC Psychiatry 2008;8:91.

53. Davidson KW, Goldstein M, Kaplan RM, et al. Evidence-based behavioral medicine: what is it and how do we achieve it? Ann Behav Med 2003;26:161-71.

54. Braun V, Clarke V. Using thematic analysis in psychology. Qual Res Psychol 2006;3:77-101.

55. Diamond IR, Grant RC, Feldman BM, et al. Defining consensus: a systematic review recommends methodologic criteria for reporting of Delphi studies. J Clin Epidemiol 2014;67:401-9.

56. Kalitzkus V, Vollmar HC. Familienmedizin in der hausarztpraxis: eine delphi-studie zur entwicklung einer gemeinsamen arbeitsdefinition. Z Allgemeinmed 2016;92:5. 